\title{
Prevalence and antimicrobial sensitivity pattern of urinary tract infection among children with cerebral palsy, Moshi, Tanzania
}

This article was published in the following Dove Press journal:

Pediatric Health, Medicine and Therapeutics

\section{Amon Ryakitimbo' \\ Rune Philemon ${ }^{1,2}$ \\ Festo Mazuguni ${ }^{3}$ \\ Levina Msuya ${ }^{1,2}$}

'Department of Pediatric and Child Health, Kilimanjaro Christian Medical University College, Moshi, Tanzania; ${ }^{2}$ Department of Pediatric and Child Health, Kilimanjaro Christian Medical Centre, Moshi, Tanzania; ${ }^{3}$ Department of Epidemiology and Applied Biostatistics, Institute of Public Health, Kilimanjaro Christian Medical University College, Moshi, Tanzania

Correspondence: Amon Ryakitimbo Department of Pediatric and Child Health, Kilimanjaro Christian Medical University College, P O Box 2240, Moshi, Tanzania

Tel +255755009998

Email amonmasangula@ymail.com
Background: Urinary tract infection (UTI) in children with cerebral palsy (CP) is a challenging yet common clinical condition. Children with $\mathrm{CP}$ bare the greatest risk of contracting UTI because of their difficulties in neuromotor control which lead to delay of bladder control, causing incomplete bladder emptying and urine retention.

Method: This was an analytical cross-sectional study that was conducted from September 2016 to March 2017 at Comprehensive Community Based Rehabilitation in Tanzania - Moshi and Kilimanjaro Christian Medical Centre Neurological Pediatrics Outpatient Clinic. All children who met the inclusion criteria were studied. Urine samples were collected at one point by catheterization, and urine dipstick and urine culture were done. Data were analyzed using SPSS version 20.

Results: A total of 99 children were enrolled in the study. The median age was 4 years (3-8 years); $53.5 \%$ were aged between 2 and 4 years. More than half were male. UTI was detected in $13.1 \%(\mathrm{n}=13)$ of the children. Five causative agents of UTI were isolated, namely Escherichia coli, Proteus mirabilis, Klebsiella pneumonia, Staphylococcus aureus, and Enterococcus faecalis. The two most common organisms, E. coli and P. mirabilis, both had low sensitivity to ampicillin and co-trimoxazole while they were sensitive to ciprofloxacin and ceftriaxone.

Conclusion: UTI is a common finding among children with CP. E. coli and P. mirabilis are the commonest causative agents and are sensitive to ciprofloxacin and ceftriaxone but have low sensitivity to ampicillin and co-trimoxazole.

Keywords: urinary tract infection, cerebral palsy, Moshi, Tanzania

\section{Introduction}

Urinary tract infection (UTI) is a challenging yet common and important clinical problem in children. ${ }^{1-3}$ A single encounter of UTI is enough to cause renal scaring, which may eventually lead to a child developing hypertension and even end-stage renal failure as long-term consequences. ${ }^{4-9}$

The factors that increase the likelihood of acquiring UTI include delay to attain bladder and bowel control, difficulty in neuromotor control of posture, low cognition, limited ability to communicate the need to void, constipation, impaired mobility, and bladder dysfunction. ${ }^{10-12}$ All these are common findings among children with cerebral palsy $(\mathrm{CP}) .^{13}$

The burden of UTIs among children with CP in the developed world ranges from $8.5 \%$ to $56.7 \%$ while in Africa, Nigeria in particular, the prevalence was found to be $38.5 \% .{ }^{14-19}$ The most common causative pathogens include Escherichia coli, Proteus 
spp, Enterococcus faecalis, Klebsiella spp, and Staphylococcus spp..$^{15,18,19}$ The recommended treatments for these pathogens are amoxiclav and co-trimoxazole. ${ }^{1}$ There are however reports of resistance to these first-line agents. ${ }^{19}$

Children with $\mathrm{CP}$ often receive empirical antibiotics for the treatment of UTI without confirmation of the presence of UTI or testing of sensitivity pattern. They also have other frequent comorbidities like upper respiratory infections and aspiration pneumonia which increase their exposure to antibiotics. ${ }^{20,21}$ This puts them at the risk of accumulating antibiotic-resistant pathogens and increasing drug-resistant strains in the community which is a major concern in light of the limited choices we have. It has been reported that children who are exposed to antibiotics have higher odds of harboring resistant pathogens compared to those who are not. ${ }^{22}$

Lack of evidence-based diagnosis and treatment also puts unnecessary costs on the families, who are often poor, to treat presumed UTIs with drugs that are not effective. We were unable to find any published studies about UTI in children with CP in Tanzania.

The aim of this study was to determine the burden of UTI, its causative organism, and the antimicrobial sensitivity pattern among children with CP in Moshi. The research will improve the knowledge of the burden of the disease and will also enable clinicians to select antibiotics that will maximize cure while decreasing complication and chances of antibiotic resistance.

\section{Materials and methods}

This was an analytical cross-sectional study conducted from September 2016 to March 2017. It was conducted at Comprehensive Community Based Rehabilitation in Tanzania (CCBRT) - Moshi and Kilimanjaro Christian Medical Centre Neurological Pediatrics Outpatient Clinic (KCMC NPOC) in Moshi. CCBRT Moshi (House of Hope) is a rehabilitation centre, located in Kaloleni, Moshi. Therapy provided is done through scheduled weeks of therapy, "week of intensive therapy (WIT)", in which children with different disabilities have a week stay at the rehabilitation centre for both rehabilitative and medical interventions.

$\mathrm{KCMC}$ is a zonal referral hospital in northern Tanzania running a weekly pediatric neurology clinic (where this study was conducted), among other inpatient and outpatient services. The study population comprised all children who had a diagnosis of $\mathrm{CP}$ attending CCBRT Moshi and KCMC NPOC for any reasons during the study period.
The study included all children aged 2-18 years who were diagnosed with CP. Children whose caregivers did not consent, those with documented use of any antibiotic within 2 weeks prior to enrollment or who had menstrual periods or any vaginal discharge were excluded. Parents/caregivers provided written informed consent for the participation of the children in the study.

A questionnaire was used to collect demographic and clinical data. Clinical examination and gross motor dysfunction classification was done by the principal investigator. The information that was collected included age, sex, address, diagnosis, gross motor function, and a short history. The parents or caregivers were asked about history of fever, blood in urine, color of urine, bladder and bowel control, and previous history of UTI or abnormalities.

The gross motor function classification system (GMFCS) was used as described by other researchers. ${ }^{23,24}$ The GMFCS is a 5-level classification that has been categorized into four age groups, which are before second birthday, between second and fourth birthday, between fourth and sixth birthday, and between sixth and twelfth birthday. It focuses on a child's self-initiation of sitting and walking activities. GMFCS levels I and II are stated as mild, level III as moderate, and levels IV and $\mathrm{V}$ as severe. ${ }^{19,23}$

\section{Urine sample collection}

The investigator examined the children and collected urine samples in two sterile bottles. The steps for urine sample collection were followed as described by Bajaj and Bothner. ${ }^{25}$ Urine samples were collected by using Folycath ${ }^{\circledR} 2$ way Foley catheter (MEDEX Healthcare, Beijing, China) size 6,8 , and 10 French depending on the age of the child. Spot urine dipstick test (Neotest ${ }^{\circledR}$ urine multistix; Neomedic Ltd, Rickmansworth, UK) was performed in one urine sample to detect nitrites and leukocyte esterase. The second sample was taken for culture and sensitivity. All samples for culture were stored in a cool box at a temperature of $5^{\circ} \mathrm{C}-7^{\circ} \mathrm{C}$ and then transported to the laboratory (KCMC Clinical Laboratory) for culture within 3 hours of collection.

\section{Laboratory methods}

Culture processing was done by inoculation of urine into MacConkey agar and 5\% blood agar medium plate by using a standard $1 \mu$ loop, to allow growth of both Gram-negative and Gram-positive organisms. The plates were incubated at $37^{\circ} \mathrm{C}$, and the number of colonies was counted at the 24th hour. Growth of a single uropathogen with at least 50,000 
colony-forming units per microliter was considered as positive culture. Bacterial colonies on solid agar were then identified based on a characteristic morphology and gram stain appearance. Biochemical identification test, Kligler iron agar, sulfur indole motility, citrate agar, oxidase, and urea were used for organism identification.

\section{Drug susceptibility testing}

Antimicrobial sensitivity testing was performed on selected antibiotics recommended by the World Health Organization and are commonly used. They included ampicillin $(10 \mu \mathrm{g})$, amoxicillin-clavulanic acid $(20 / 10 \mu \mathrm{g})$, nitrofurantoin, cotrimoxazole $(30 \mu \mathrm{g})$, gentamicin $(10 \mu \mathrm{g})$, ciprofloxacin $(5$ $\mu \mathrm{g})$, cefotaxime $(30 \mu \mathrm{g})$, and ceftriaxone $(30 \mu \mathrm{g})$. The testing was done by Kirby-Bauer diffusion method by using Muller Hinton agar which was then incubated for 18-24 hours at $37^{\circ} \mathrm{C}$. Antimicrobial sensitivity was reported as resistant, intermediate, and sensitive according to Clinical Laboratory Standard Institute. ${ }^{26}$

\section{Ethical clearance}

Ethical clearance was obtained from the Kilimanjaro Christian Medical University College Research Ethics Committee and certificate number 968 was granted, and those who did not participate received equal care.

\section{Data analysis}

Data were coded and entered into SPSS version 20 (IBM Corporation, Armonk, NY, USA). Descriptive statistics were summarized by frequency and proportions. Continuous data were summarized as median along with interquartile range (IQR) values. Test for association between dependent and independent categorical variables was carried by using chisquare test. $P$-value of $\leq 0.05$ was considered significant.

\section{Results}

\section{Demographic characteristics of the study participants}

A total of 107 eligible children were recruited between September 2016 and March 2017. Participants from CCBRT Moshi and KCMCNPOC were 100 and 7, respectively. Ninety-nine children were included in the final analysis. Eight children were excluded because they could not tolerate catheterization (Figure 1).

A majority of the children (53.5\%) were aged between 2 and 4 years. The median (IQR) age at enrollment was 4 years (range $=3-8$ years). Male gender constituted a higher proportion $(58.6 \%)$ (Table 1$)$.

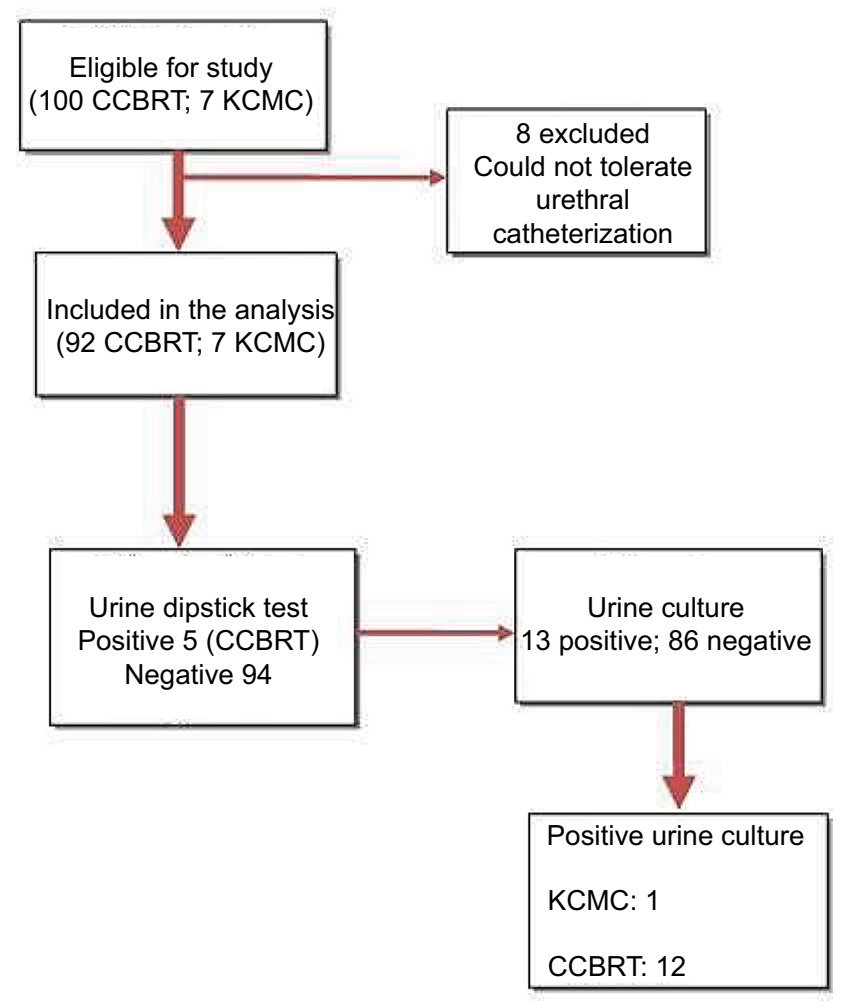

Figure I Study flow diagram.

Abbreviations: CCBRT, Comprehensive Community Based Rehabilitation in Tanzania; KCMC, Kilimanjaro Christian Medical Centre.

Table I Demographic characteristics of the study participants $(\mathrm{N}=99)$

\begin{tabular}{ll}
\hline Characteristics & $\mathbf{n}(\%)$ \\
\hline $\begin{array}{l}\text { Median age (IQR), years } \\
\text { Age, years } \\
2-4\end{array}$ & $4(3-8)$ \\
$5-7$ & $53(53.5)$ \\
$8-10$ & $21(21.2)$ \\
$\geq I 1$ & $16(16.2)$ \\
Sex & $9(9.1)$ \\
Male & $58(58.6)$ \\
Female & $41(41.4)$ \\
Residence & \\
Urban & $50(50.5)$ \\
Rural & $37(37.4)$ \\
Other & $12(12.1)$ \\
\hline
\end{tabular}

Abbreviation: IQR, interquartile range.

\section{Clinical characteristics of the study participants}

Those with a spastic quadriplegic type of $\mathrm{CP}$ constituted $64.6 \%$ of the children followed by hemiplegic CP $(17.2 \%)$, athetoid $\mathrm{CP}(7.1 \%)$, mixed type $\mathrm{CP}(6.1 \%)$, diplegic $\mathrm{CP}(4 \%)$, and dystonic type of CP (1\%). None had ataxic type of CP.

About $34 \%(n=34)$ of the study children had mild motor dysfunction, including $26.3 \%(\mathrm{n}=26)$ with grade II and $8.1 \%$ 
$(n=8)$ with grade I. Moderate motor dysfunction was seen in $19.2 \%(n=19)$. Severe motor dysfunction was seen in $46.5 \%$ $(n=46)$ of children; of these $25.3 \%(n=25)$ had grade IV and $21.2 \%(n=21)$ had grade $V$.

\section{Symptoms and signs of UTI}

Most (95\%) of the interviewed caregivers or parents could not provide information regarding some of the UTI symptoms. The symptoms included frequency of urination, urgency, abdominal pain, and pain or straining during micturition. They reported that it was hard to ascertain because of their child's mental condition.

Information readily obtained from the caregivers included previous history of use of antibiotics, incontinence, constipation, fever, and smell and color of urine. About $18.1 \%$ had a history of using antibiotics at least 2 weeks before attending the rehabilitation center due to various reasons. Three of them were admitted due to respiratory infection and treated with intravenous drugs, probably antibiotics. None of them reported that they had malaria. The other 15 children were treated as outpatients. The antibiotics prescribed included amoxicillin, metronidazole, cephalexin, co-trimoxazole, and erythromycin. There was only one positive urine culture among those with a history of using antibiotics $>2$ weeks prior to enrollment.

About $14.1 \%$ had a previous history of UTI within 2 years prior to enrollment; the diagnosis was clinical, and they were treated with antibiotics. Of these, only one had a positive urine culture.

Regarding bladder control, among our study participants, $75.8 \%$ had urine incontinence, $18.2 \%$ were continent, and $6 \%$ either had day or night incontinence. Among those with incontinence, eight had positive urine cultures.

Other clinical information gathered in relation to UTI included constipation (less than three bowel motions in a week) which was reported among $30.3 \%$ of the participants. Over two thirds (71.7\%) had cloudy and smelly urine. Among those with constipation and cloudy and smelly urine, there were four and 11 positive urine cultures, respectively. On examination, five children had temperatures above $37.5^{\circ} \mathrm{C}$.

One female child tested positive for leukocyte esterase on urine dipstick with no nitrites. However, the urine culture and sensitivity analysis showed no growth. Three had aspiration pneumonia and were treated with ampiclox syrup plus metronidazole. Carbuncle was seen in one child, and she was given ampiclox syrup. All had recovered well upon followup. The clinical characteristics of the study participants are summarized in Table 2.
Table 2 Clinical characteristics of the study participants $(\mathrm{N}=99)$

\begin{tabular}{|c|c|}
\hline Characteristics & $\mathbf{N}(\%)$ \\
\hline \multicolumn{2}{|l|}{ Weight (kg) } \\
\hline$<10$ & $34(34.3)$ \\
\hline $11-20$ & $50(50.5)$ \\
\hline$>20$ & $15(15.2)$ \\
\hline \multicolumn{2}{|c|}{ Types of cerebral palsy } \\
\hline \multicolumn{2}{|c|}{ Spastic cerebral palsy } \\
\hline Diplegia & $4(4.0)$ \\
\hline Hemiplegia & $17(17.2)$ \\
\hline Quadriplegia & $64(64.6)$ \\
\hline Athetoid & $7(7.1)$ \\
\hline Dystonia & $\mathrm{I}(\mathrm{I} .0)$ \\
\hline Mixed type & $6(6.1)$ \\
\hline \multicolumn{2}{|c|}{ Gross motor function } \\
\hline Mild & $34(34.3)$ \\
\hline Moderate & $19(19.2)$ \\
\hline Severe & $46(46.5)$ \\
\hline \multicolumn{2}{|c|}{ Urine is smelly and cloudy } \\
\hline Yes & $7 \mid(71.7)$ \\
\hline No & $28(28.3)$ \\
\hline \multicolumn{2}{|c|}{ Pattern of continence } \\
\hline Continent & $18(18.2)$ \\
\hline Nocturnal only & $2(2.0)$ \\
\hline Diurnal only & $4(4.0)$ \\
\hline Incontinent & $75(75.8)$ \\
\hline \multicolumn{2}{|l|}{ Constipation } \\
\hline Yes & $30(30.3)$ \\
\hline No & $69(69.7)$ \\
\hline \multicolumn{2}{|c|}{ Previous history of urinary tract infection } \\
\hline Yes & $14(14.1)$ \\
\hline No & $85(85.9)$ \\
\hline \multicolumn{2}{|l|}{ Fever } \\
\hline Yes & $5(5.1)$ \\
\hline No & $94(94.9)$ \\
\hline \multicolumn{2}{|c|}{ Previous history of antibiotic use } \\
\hline \multicolumn{2}{|c|}{2 weeks + prior rehabilitation* } \\
\hline Yes & $18(18.1)$ \\
\hline No & $81(81.9)$ \\
\hline
\end{tabular}

Note: *Amoxicillin, metronidazole, cephalexin, co-trimoxazole, and erythromycin.

\section{Prevalence of UTI}

Urine culture was used as the confirmatory test in this study. The prevalence of UTI among children with CP attending CCBRT Moshi and KCMC NPOC was 13.1\%. A few (5.1\%) samples were leukocyte esterase positive and nitrite negative. None of the samples had either a positive nitrite or positive of both leukocyte esterase and nitrites.

There was no demographic or clinical characteristic which was associated with UTI among the study children (chi-square $p$-value $>0.05$ ) (Table 3 ).

\section{Etiological agents}

Five pathogens were isolated from 13 positive urine cultures. E. coli was the most common isolated pathogen 
Table 3 Association between some characteristics of participants with cerebral palsy and presence of urinary tract infection (UTI)

\begin{tabular}{|c|c|c|c|c|}
\hline Characteristics & Total & UTI, n (\%) & $\chi^{2}$ & $P$-value \\
\hline \multicolumn{5}{|l|}{ Age range, years } \\
\hline $2-4$ & 53 & $9(17.0)$ & & \\
\hline $5-7$ & 21 & $3(14.3)$ & & \\
\hline $8-10$ & 16 & I (6.2) & & \\
\hline$>11$ & 9 & $0(0.0)$ & 2.738 & 0.434 \\
\hline \multicolumn{5}{|l|}{ Sex } \\
\hline Male & 58 & $8(13.8)$ & & \\
\hline Female & 41 & $5(12.2)$ & 0.054 & 0.817 \\
\hline \multicolumn{5}{|l|}{ Temperature } \\
\hline Fever & 5 & $2(40.0)$ & & \\
\hline No fever & 94 & $11(11.7)$ & 3.333 & 0.068 \\
\hline \multicolumn{5}{|c|}{ Gross motor function } \\
\hline Mild & 34 & $5(14.7)$ & & \\
\hline Moderate & 19 & $\mathrm{I}(5.3)$ & & \\
\hline Severe & 46 & $7(\mid 5.2)$ & 1.281 & 0.527 \\
\hline \multicolumn{5}{|c|}{ Urine smell and color } \\
\hline Yes & 28 & $2(7.1)$ & & \\
\hline No & 71 & II (I5.5) & 1.227 & 0.268 \\
\hline \multicolumn{5}{|l|}{ Continence } \\
\hline Continent & 18 & $3(16.7)$ & & \\
\hline Nocturnal only & 2 & I $(50.0)$ & & \\
\hline Diurnal only & 4 & I $(25.0)$ & & \\
\hline Incontinent & 75 & $8(10.7)$ & 3.474 & 0.324 \\
\hline \multicolumn{5}{|l|}{ Constipation } \\
\hline Yes & 30 & $4(13.3)$ & & \\
\hline No & 69 & $9(13.0)$ & 0.002 & 0.969 \\
\hline \multicolumn{5}{|c|}{ Previous history of UTI } \\
\hline Yes & 14 & I (7.I) & & \\
\hline No & 85 & $12(14.1)$ & 0.513 & 0.474 \\
\hline \multicolumn{5}{|l|}{ Medications } \\
\hline \multicolumn{5}{|l|}{ taken for UTI } \\
\hline Yes & 18 & $\mathrm{I}(5.6)$ & & \\
\hline No & 81 & $13(13.1 \%)$ & 1.107 & 0.293 \\
\hline
\end{tabular}

found in seven $(53.8 \%)$ children. Others included Proteus mirabilis, identified from three (23.1\%) children, Klebsiella pneumoniae from one (7.7\%) child, and two Gram-positive organisms, Staphylococcus aureus and E. faecalis, from two children.

\section{Antimicrobial drug sensitivity pattern}

$E$. coli isolated from the urine of seven children was least susceptible to co-trimoxazole $(28.5 \%)$ and ampicillin (28.5\%). P. mirabilis was also least susceptible to the same antibiotics. E. coli was $100 \%$ sensitive to ciprofloxacin and ceftriaxone but was less sensitive to amoxicillin-clavulanic acid, nitrofurantoin, gentamicin, and cefotaxime (71\%-85\%). P. mirabilis showed a similar trend. There was no mixed growth or contaminants (Table 4).

\section{Discussion}

The prevalence of UTI among children with CP in Tanzania was $13.1 \%$. This was similar to studies performed in Turkey. ${ }^{14,18}$ It was lower compared to the majority of studies conducted elsewhere. ${ }^{15-17,19}$ The causative organisms of UTI and their sensitivity patterns were similar to previous studies. ${ }^{15,18,19}$ The similarities in findings observed in the current study and the few conducted in Turkey can be attributed to them sharing similar study designs and inclusion of young children with CP regardless of their clinical symptoms, just like the current study.

The majority of the studies done previously documented a higher proportion of UTI compared to the current study. They included older children (mean age 8 years) with fewer urinary tract symptoms and they also collected urine samples by clean catch method. ${ }^{15,17}$

Silva et $\mathrm{al}^{16}$ found that $56.7 \%$ of the children had UTI, which is a huge disparity with the current study. This can be explained by the small sample size $(\mathrm{N}=37)$, and the characteristics of the participants studied by Silva et al. A majority of the participants were female with moderate to severe motor dysfunction using wheelchairs and they had fewer urinary tract symptoms, although the mode of urine collection was not stated.

In the African setting, only one study has been published on UTI in CP. Anígilájé and Bitto ${ }^{19}$ in Nigeria reported a higher prevalance $(38.5 \%)$ compared to that of the current

Table 4 Antimicrobial drug sensitivity patterns of the isolated organisms $(n=13)$

\begin{tabular}{|c|c|c|c|c|c|}
\hline \multirow[t]{2}{*}{ Antimicrobial drug } & \multicolumn{5}{|c|}{ Isolated organism n (\%)* } \\
\hline & Escherichia coli & Proteus mirabilis & Klebsiella pneumoniae & Staphylococcus aureus & Enterococcus faecalis \\
\hline Cefotaxime & $5(7 I)$ & I (33.3) & I (I00) & I (I00) & I (100) \\
\hline Ciprofloxacin & $7(100)$ & $3(100)$ & I (100) & I (100) & I (100) \\
\hline Gentamicin & $6(85)$ & $3(100)$ & I (100) & I (I00) & I (I00) \\
\hline Nitrofurantoin & $6(85)$ & I (33.3) & $0(0.0)$ & I (100) & I (I00) \\
\hline Amoxicillin-clavulanic acid & $6(85)$ & $2(66.6)$ & $I(100)$ & $I(100)$ & I (100) \\
\hline Ceftriaxone & $7(100)$ & $3(100)$ & I (I00) & I (I00) & I (I00) \\
\hline Ampicillin & $2(28.6)$ & $0(0.0)$ & $0(0.0)$ & $0(0.0)$ & I (100) \\
\hline Co-trimoxazole & $2(28.6)$ & I (33.3) & $0(0.0)$ & I (100) & I (I00) \\
\hline
\end{tabular}

Note: $*_{n}(\%)$ represents number (\%) of sensitivity for each isolated organism. 
study because of the difference in mode of urine sample collection and where it was collected. The observed prevalance might have been due to contamination from the genitalia or perineum and not organisms from the bladder, as urine sample collection was done at home and only routine personal hygiene was required. The time lapse between the time of urine sample collection and the testing of the urine was not accounted for. To minimize false positives, it is recommended that urine analysis should be done on a fresh urine sample at room temperature in less than an hour after its collection. ${ }^{1,27}$ In the current study, urine samples were collected by sterile transurethral catheterization. This minimized the chances of contamination.

Also, the observed prevalence in the current study could be influenced by the use of antibiotics at least 2 weeks before attending the rehabilitation center, due to various reasons that were reported by 18 of the respondents, The most used antibiotic was amoxicillin; this could have cleared off any organisms that could be isolated as it was observed in a previous study. ${ }^{28}$

In the current study, no factors were associated with UTI. This is different from the study by Anígilájé and Bitto ${ }^{19}$ who found that motor disability and constipation had association with UTI. Despite the absence of association, motor function, incontinence, and constipation are of clinical significance.

Incontinence is common among children with $\mathrm{CP}$ who have moderate to severe motor impairment. In the present study, two thirds of the children $(75.8 \%)$ were incontinent, majority being male. This is similar to studies performed elsewhere. ${ }^{16,17,28}$ The higher proportion of incontinence observed in this study can be explained by the presence of a higher percentage of children aged between 2 and 4 years (53.5\%) who are not bladder trained. Children with CP attain spontaneous bladder control at around 47 months of age. ${ }^{15}$

Constipation has been demonstrated to have impact on UTI. ${ }^{30}$ In children with motor disability, a full rectum compresses the bladder and causes bladder dysfunction which leads to incomplete bladder emptying and increase in residual urine volume. Constipation occurs in $27 \%-50 \%$ of children with CP. ${ }^{16,17,19,29,30}$ In the current study, 30.3\% had constipation. This proportion might not be a true representation due to unawareness of constipation as a problem leading to possible underreporting, as has been observed by Halachmi and Farhat. ${ }^{31}$

A majority of children in our study had cloudy and turbid urine. There was no association between UTI and smelly or turbid urine, which is a similar finding to Struthers et al. ${ }^{32}$ The observed high proportion of those with smelly and turbid urine is attributed to more than half of the participants having moderate to severe motor function and depending entirely on someone else to help them drink, feed, and sometimes even taking them to the toilet. They might be getting less fluid than required. ${ }^{33}$

In the etiology of UTI in children with CP, Gram-negative organisms predominated. E. coli and P. mirabilis were the most common causative agents. The other was $K$. pneumoniae. The Gram-positive agents isolated were $S$. aureus and E. faecalis. These findings are similar to those of previous studies. ${ }^{15,18,19}$

The current study adds on the finding that E. coli in UTI is highly resistant to first-line antibiotics, co-trimoxazole and ampicillin, and is sensitive to ciprofloxacin and ceftriaxone, as has been found by other studies. ${ }^{19,22,28}$ We rarely use quinolones in children, and ceftriaxone is used in hospitals when there is severe infection. Exposure to the first-line antibiotics is however frequent as they are commonly given in primary health facilities and can even be procured over the counter. The observed resistance to first-line antibiotics may be explained by inappropriate use of these antibiotics, that is either patients use low doses or they do not adhere to the prescriptions, if any are provided. This observation is alarming, necessitating a call for local epidemiological surveillance of the antibiotic resistance patterns in our setting.

Generalization of the findings may not be plausible because of the minimal representation of older children. Also, there could be underreporting of antibiotic use. This was a strict exclusion criterion, and blood drug levels were not measured.

\section{Conclusion}

The prevalence of UTI among children with $\mathrm{CP}$ attending CCBRT Moshi and KCMC NPOC was 13.1\%. E. coli and P. mirabilis were found to be the common causative agent of UTI among children with CP in this study. Both E. coli and P. mirabilis have low sensitivity to the first-line antibiotics used; ampicillin and co-trimoxazole. Their sensitivity pattern to other antibiotics is of a varying degree.

\section{Acknowledgments}

This work was completed with support from CCBRT Moshi Team, patients and their parents/caretakers, Pediatric and Child Health Department, and KCMC Clinical Laboratory. Thanks to Dr Grace Kinabo, Dr Annette Baine, Dr Christina Kindole, Dr Angelika Masao, Dr Rose Chengo, Dr Esther Majaliwa, and Dr Grace Mella for their support. 


\section{Disclosure}

The authors report no conflicts of interest in this work.

\section{References}

1. Roberts KB. Urinary tract infection: clinical practice guideline for the diagnosis and management of the initial UTI in febrile infants and children 2 to 24 months. Pediatrics. 2011;128(3):595-610.

2. Finnell SME. Urinary tract infection in children : an update. Open Urol Nephrol J. 2015;8:92-95.

3. Hanna D, Voort JV. Childhood urinary tract infections : an evidencebased approach. Paediatr Child Health. 2016;26(8):328-332.

4. Coulthard MG, Lambert HJ, Keir MJ. Occurrence of renal scars in children after their first referral for urinary tract infection. $B M J$. 1997;315(7113):918-919.

5. Faust WC, Diaz M, Pohl HG. Incidence of post-pyelonephritic renal scarring : a meta-analysis of the dimercapto-succinic acid literature. $J$ Urol. 2009;181(1):290-297.

6. Shaikh N, Ewing AL, Bhatnagar S, Hoberman A. Risk of renal scarring in children with a first urinary tract infection : a systematic review. Pediatrics. 2010;126(6):1084-1091.

7. Park YS. Renal scar formation after urinary tract infection in children. Korean J Paediatr. 2012;55(10):367-370.

8. Coulthard MG, Lambert HJ, Vernon SJ, Hunter EW, Keir MJ, Matthews JNS. Does prompt treatment of urinary tract infection in preschool children prevent renal scarring: mixed retrospective and prospective audits. Arch Dis Child. 2014;99(4):342-347.

9. Shaikh N, Craig JC, Rovers MM, et al. Identification of children and adolescents at risk for renal scarring after a first urinary tract infection: a meta-analysis with individual patient data. JAMA Pediatr. 2014;15224(10):893-900.

10. Bhat RG, Katy TA, Place FC. Pediatric urinary tract infections. Emerg Med Clin North Am. 2011;29(3):637-653.

11. Becknell B, Schober M, Korbel L, Spencer JD. The diagnosis, evaluation and treatment of acute and reccurent pediatric urinary tract infections. Expert Rev Anti Infect Ther. 2015;13(1):81-90.

12. Keren R, Shaikh N, Pohl H, et al. Risk factors for recurrent urinary tract infection and renal scarring. Pediatrics. 2015;136(1):e13-e21.

13. Pakula AT, Braun KVN. Cerebral palsy: classification and epidemiology. Phys Med Rehabil Clin N Am. 2009;20(3):425-452.

14. Karaman MI, Kaya C, Caskurlu T, Guney S, Ergenekon E. Urodynamic findings in children with cerebral palsy. Int J Urol. 2005;12(8): 717-720.

15. Ozturk M, Oktem F, Kisioglu N, Demirci M, Altuntas I. Bladder and bowel control in children with cerebral palsy: case-control study. Croat Med J. 2006;47(2):264-270.

16. Silva AF, Alvares RA, Barboza L, Tempora R. Lower urinary tract dysfunction in children with cerebral palsy. Neurol Urodynamics. 2009;28(8):959-963.
17. Silva JAF, Gonsalves MCD, Saverio AP, Oliveira IC, Carrerette FB, Damião R. Lower urinary tract dysfunction and ultrasound assessment of bladder wall thickness in children with cerebral palsy. Pediatr Urol. 2010;76(4):942-945.

18. Özen M, Güngör S, Raif SG. The overlooked childhood problems in pediatric cerebral palsy subjects. JIUMF. 2012;19(1):1-5.

19. Anígilájé EA, Bitto TT. Prevalence and predictors of urinary tract infections among children with cerebral palsy in Makurdi, Nigeria. Int J Nephrol. 2013;2013:937268.

20. Young NL, Mccormick AM, Gilbert T, et al. Reasons for hospital admissions among youth and young adults with cerebral palsy. Arch Phys Med Rehabil. 2011;92(1):46-50.

21. Fahimzad A, Babaie D, Ghoroubi J, Zahed G, Tabatabaei SR. Common infections among disabled children admitted to hospital. Arch Pediatr Infect Dis. 2013;1(2):71-74.

22. Bryce A, Hay AD, Lane IF, Thornton HV, Wootton M, Costelloe C. Global prevalence of antibiotic resistance in paediatric urinary tract infections caused by Escherichia coli and association with routine use of antibiotics in primary care : systematic review and meta-analysis. BMJ. 2016;352:1939.

23. Palisano R, Rosenbaum P, Walter S, Russel D, Wood E, Galuppi B Development and reliability of a system to classify gross motor function in children with cerebral palsy. Dev Med Child Neurol. 1997;39(2):214-223.

24. Krigger KW. Cerebral palsy: an overview. Am Fam Physician. 2006;73(1): 91-100.

25. Bajaj L, Bothner J. Urine collection techniques in children. In: Stack AM, Wiley JF, editors. UpToDate ${ }^{\circledR}$. 2016. Available from: https://www. uptodate.com. Accessed August 13, 2016

26. Clinical Laboratory Standard Institute. Performance Standards for Antimicrobial Susceptibility Testing; Twenty-Second Informational Supplement. 2012. Available from http://www.facm.ucl.ac.be. Accessed August 13, 2016.

27. Utsch B, Klaus G. Urinalysis in children and adolescents. Dtsch Ärzteblatt Int. 2014;111(37):617-625.

28. Gidabayda JG, Philemon R, Abdallah MS, et al. Patterns of urinary tract infection amongst children admitted at Kilimanjaro Christian Medical Centre. East African Heal Res J. 2017;1(1):53-61.

29. Gundogdu G, Komur M, Alvan D, et al. Relationship of bladder dysfunction with upper urinary tract deterioration in cerebral palsy. $J$ Pediatr Urol. 2013;9(5):659-664.

30. Hoque SA, Islam T, Ahmed F, Hanif M. Impact of constipation in children on urinary tract infection (UTI). Bangladesh $J$ Child Heal. 2010;34(1):17-20.

31. Halachmi S, Farhat WA. Interactions of constipation, dysfunctional elimination syndrome, and vesicoureteral reflux. Adv in Urol. 2008;2008:828275.

32. Struthers S, Scanlon J, Parker K, Goddard J, Hallett R. Parental reporting of smelly urine and urinary tract infection. Arch Dis Child. 2003;88:250-252.

33. Van Laecke E, Raes A, Vande Walle J, Hoebeke P. Adequate fluid intake, urinary incontinence, and physical and/or intellectual disability. J Urol. 2009;182(4):2079-2084.
Pediatric Health, Medicine and Therapeutics

\section{Publish your work in this journal}

Pediatric Health, Medicine and Therapeutics is an international, peerreviewed, open access journal publishing original research, reports, editorials, reviews and commentaries. All aspects of health maintenance, preventative measures and disease treatment interventions are addressed within the journal. Practitioners from all disciplines are invited to submit

\section{Dovepress}

their work as well as healthcare researchers and patient support groups. The manuscript management system is completely online and includes a very quick and fair peer-review system. Visit http://www.dovepress.com/ testimonials.php to read real quotes from published authors. 